\title{
Assessing the Anti-inflammatory Mechanism of Reduning Injection by Network Pharmacology
}

\author{
Fuda Xie $\mathbb{D}^{1,2}$ Mingxiang Xie, ${ }^{1,2}$ Yibing Yang, ${ }^{1,2}$ Miaomiao Zhang, ${ }^{3}$ Xiaojie Xu, ${ }^{4}$ Na Liu, ${ }^{1,2}$ \\ Wei Xiao $\mathbb{D}^{5},{ }^{5}$ and Jiangyong $G u \mathbb{D}^{1,2}$ \\ ${ }^{1}$ Research Center of Integrative Medicine, School of Basic Medical Science, Guangzhou University of Chinese Medicine, \\ Guangzhou 510006, China \\ ${ }^{2}$ Department of Biochemistry, School of Basic Medical Science, Guangzhou University of Chinese Medicine, \\ Guangzhou 510006, China \\ ${ }^{3}$ The Second Clinical College, Guangzhou University of Chinese Medicine, Guangzhou 510006, China \\ ${ }^{4}$ College of Chemistry and Molecular Engineering, Peking University, Beijing 100871, China \\ ${ }^{5}$ State Key Laboratory of New-Tech for Chinese Medicine Pharmaceutical Process, Lianyungang 222001, China
}

Correspondence should be addressed to Wei Xiao; xw_kanion@163.com and Jiangyong Gu; gujy@gzucm.edu.cn

Received 15 June 2020; Revised 30 October 2020; Accepted 4 December 2020; Published 16 December 2020

Academic Editor: Bing Wang

Copyright ( 2020 Fuda Xie et al. This is an open access article distributed under the Creative Commons Attribution License, which permits unrestricted use, distribution, and reproduction in any medium, provided the original work is properly cited.

\begin{abstract}
Reduning Injection (RDNI) is a traditional Chinese medicine formula indicated for the treatment of inflammatory diseases. However, the molecular mechanism of RDNI is unclear. The information of RDNI ingredients was collected from previous studies. Targets of them were obtained by data mining and molecular docking. The information of targets and related pathways was collected in UniProt and KEGG. Networks were constructed and analyzed by Cytoscape to identify key compounds, targets, and pathways. Data mining and molecular docking identified 11 compounds, 84 targets, and 201 pathways that are related to the anti-inflammatory activity of RDNI. Network analysis identified two key compounds (caffeic acid and ferulic acid), five key targets (Bcl-2, eNOS, PTGS2, PPARA, and MMPs), and four key pathways (estrogen signaling pathway, PI3K-AKT signaling pathway, cGMP-PKG signaling pathway, and calcium signaling pathway) which would play critical roles in the treatment of inflammatory diseases by RDNI. The cross-talks among pathways provided a deeper understanding of anti-inflammatory effect of RDNI. RDNI is capable of regulating multiple biological processes and treating inflammation at a systems level. Network pharmacology is a practical approach to explore the therapeutic mechanism of TCM for complex disease.
\end{abstract}

\section{Introduction}

Inflammation is regarded as a kind of congenital immunity as well as the basis of various physiological and pathological processes, and it can affect human health and living quality in many respects [1-4]. Five typical symptoms of inflammation are fever, pain, redness, swelling, and loss of function [5]. Acute inflammation is the body's initial response to harmful stimuli such as burns, pathogen infection, and toxins [6, 7]. Chronic inflammation is a biological progress that leads to multiple diseases such as hay fever, periodontitis, atherosclerosis, rheumatoid arthritis, and even cancer [8-10]. There are at present more than one hundred FDA-approved anti- inflammatory drugs, which are classified into steroid and nonsteroidal anti-inflammatory drugs [11-13]. However, these drugs are related to various side effects including irreversible sensor neural hearing loss [14], gastrointestinal symptoms (such as dyspepsia, gastrointestinal bleeds, or even gastrointestinal perforations) [15], and side effects on cartilage metabolism. [16]

Traditional Chinese medicines (TCMs) have been used to treat various diseases including inflammation for a long time. For example, Reduning Injection [17], Bi-Qi capsule [18], and Shuanghuanglian injection [19] are widely used in China for treating inflammation. The validity and safety of these TCM formulas are already verified by thousands of years of 
clinical applications. Some of them have been studied in modern approaches, and their effectiveness and molecular mechanism were demonstrated by the results $[17,20,21]$. TCM can regulate multiple pathogenic progresses so as to cure diseases effectively and completely in a holistic manner $[19,20,22]$. However, the ingredients of TCM are complicated and can interact with multiple targets. It is difficult to elucidate the mechanism of the action (MOA) of TCM by traditional pharmacological methods.

Network pharmacology provides frameworks to understand how regulation arises from the interactions between cellular components, and it is considered the next paradigm in drug development [23]. By using network pharmacology approaches, we can build complex networks on the basis of disease-related biological progresses and adopt network analysis to obtain insights into the pharmacological mechanism. These methods can provide theoretical basis and guidance for the development of multitarget drugs, and they have been used to investigate the pathogenesis of several diseases [22, 24, 25]. Reduning Injection (RDNI) is used for the treatment of inflammatory diseases, such as upper respiratory tract infection and acute bronchitis [26-28], while the molecular mechanism of its therapeutic function is unclear. Three herbs contained in RDNI are wildly used to cure inflammation-related diseases, namely, Lonicera japonica Thunb. (honeysuckle, Jinyinhua), Gardenia jasminoides Ellis. (cape jasmine, Zhizi), and Artemisia annua L. (sweet wormwood, Qinghao) [29-31]. In our previous works, the main ingredients of RDNI and their activities against inflammation have been explored [17, 22, 27]. In this work, data mining and molecular docking were used to predict the targets of RDNI compounds and their metabolites. A compoundtarget network and a target-pathway network were constructed. Key targets and pathways were identified by network analysis and literature consulting. The cross-talks between inflammation-related pathways were also discussed. The results indicate that the molecular mechanism of the anti-inflammatory function of RDNI can be discovered by computational modeling, which provides a practical approach to study the MOA of TCM prescription.

\section{Methods}

2.1. Collection of RDNI Compounds. Nine ingredients with measurable content of RDNI have been identified in previous works [32-38]. Four metabolites of these ingredients were gathered by literature mining [39-41]. The information and 3D structures of these 13 compounds were downloaded from PubChem (http://pubchem.ncbi.nlm.nih.gov) [42], a chemical database of authoritative sources.

2.2. Target Mining. The targets of RDNI compounds and metabolites were collected by database searching and molecular docking. Four databases were used in this step, namely, PubChem, Traditional Chinese Medicine Systems Pharmacology Database and Analysis Platform (TCMSP, http://sp .nwu.edu.cn/tcmsp.php) [43], Binding DataBase (BindingDB, http://www.bindingdb.org/bind/index.jsp) [44, 45], and DrugBank (http://www.drugbank.ca) [46]. TCMSP is a systems pharmacology platform that provides the relationships between Chinese herbal medicines and their targets. BindingDB can provide measured binding affinities between compounds and their targets. First, CID codes in PubChem were used to find the records of RDNI compounds, and their targets were obtained in the "Biological Test Results." Second, the records of compounds in TCMSP were retrieved by the CAS registry number, and the targets' information was collected from the "Related Targets" section. Finally, the tool "Find my Compound's Target" in BindingDB was used to screen targets of RDNI compounds. Targets gathered in PubChem, BindingDB, and TCMSP are recorded in Supplementary Table S1.

The DrugBank database can provide detailed drug data and comprehensive drug target information. There are 126 FDA-approved anti-inflammatory drugs in DrugBank [46]. Their targets were collected, and known protein structures were downloaded from the RSCB protein data bank (http://www.rcsb.org). Molecular docking was adopted to evaluate the binding affinity between each compound and target by Autodock 4.2.6 [47]. The energy grid was a 30 $\times 30 \times 30 \AA$ cube centered on the occupied space of the original ligand with a spacing of $0.375 \AA$ between the grid points. The Lamarckian genetic algorithm (LGA) was used to optimize the conformation of compound in the binding pocket. The parameters for LGA were listed as follows: the number of individuals in population, maximum number of energy evaluations, and the maximum number of generations, and the rate of gene mutation was set as 150, 2.5 $\times 10^{6}, 2.7 \times 10^{4}$, and 0.02 , respectively. Other parameters were set to default. The docking results were sorted according to the binding energy, and the proteins with binding energy lower than $-8.18 \mathrm{kcal} / \mathrm{mol}$ (the threshold for inhibition constant was $1 \mu \mathrm{M}$ ) were regarded as a target of the corresponding compound.

2.3. Network Construction. On the basis of compoundtarget interaction obtained in the previous step, the compound-target network (CTN) was constructed and visualized by Cytoscape version 3.6.1 [48]. Key targets and main active components of RDNI for treating inflammation were predicted by degree centrality, betweenness centrality, and closeness centrality. These network topological parameters were calculated by the NetworkAnalyzer plugin [49]. Targets that meet two screening criteria were regarded as important targets: their degree centralities were in the top ten of all involved targets; their betweenness centralities and closeness centralities were both larger than the average value of all involved targets in CTN (Supplementary Table S2). Key targets were selected from these important targets after assessment of their locations in different pathways and their regulation relationships with upstream and downstream targets. The molecular functions and related biological processes of targets were retrieved from UniProt (http://www.uniprot.org) [50].

The related pathways (Supplementary Table S3) of RDNI targets were collected from the Kyoto Encyclopedia of Genes and Genomes (KEGG, http://www.kegg.jp) [51]. The target- 
TABLE 1: Ingredients and metabolites of RDNI.

\begin{tabular}{|c|c|c|c|c|c|}
\hline Compound & Abbreviation & Content in injection $(\mathrm{mg} / \mathrm{ml})$ & MW & CID & CAS \\
\hline Neochlorogenic acid & 5CQA & 2.51 & 354.31 & 5280633 & $906-33-2$ \\
\hline Chlorogenic acid & CGA & 4.36 & 354.31 & 1794427 & $327-97-9$ \\
\hline Cryptochlorogenic acid & 4CQA & 2.01 & 354.31 & 9798666 & $905-99-7$ \\
\hline Caffeic acid & CAA & 0.09 & 180.15 & 689043 & $331-39-5$ \\
\hline Isochlorogenic acid B & IsoB & 0.33 & 516.45 & 5281780 & $14534-61-3$ \\
\hline Isochlorogenic acid $\mathrm{A}$ & IsoA & 0.19 & 516.45 & 6474310 & $89919-62-0$ \\
\hline Isochlorogenic acid C & IsoC & 0.34 & 516.45 & 6474309 & $57378-72-0$ \\
\hline Geniposide & Gen & 8.16 & 404.36 & 107848 & $169799-41-1$ \\
\hline Secoxyloganin & Sec & 0.81 & 404.36 & 162868 & $58822-47-2$ \\
\hline Ferulic acid* & FA & NA & 194.18 & 445858 & $537-98-4$ \\
\hline Dihydroferulic acid* & DFA & NA & 196.20 & 17865499 & NA \\
\hline Genipin* & Gep & NA & 226.22 & 442424 & $6902-77-8$ \\
\hline $3^{\prime}$-Hydroxycinnamic acid* & NA & NA & 164.16 & 637541 & $588-30-7$ \\
\hline
\end{tabular}

${ }^{*}$ Ferulic acid, dihydroferulic acid, genipin, and $3^{\prime}$-hydroxycinnamic acid are metabolite of caffeic acid, caffeic acid, geniposide, and caffeic acid, respectively.

pathway network (TPN) was then constructed and visualized by Cytoscape. Key pathways of RDNI were obtained on the basis of TPN and literature consulting. Twelve pathways were found to have close relationships with inflammation, and they also had high degree centralities $(>6)$ as well as high betweenness centralities (>average value) in the TPN. Four of the twelve pathways were excluded because they do not represent a specific biological process. Another two pathways were excluded because they do not have high closeness centralities (>average value) (Supplementary Table S4). The remaining six pathways were regarded as important pathways of RDNI, and a compound-targetpathway network (CTPN) was constructed by Cytoscape. Four key pathways were selected from important pathways for their close relationships with inflammatory processes. Graphs of key pathways were downloaded from KEGG. Targets of RDNI were marked in red by KEGG mapping tools.

A cross-talk network was constructed to visualize the cross-talks among key pathways and other three pathways, which were closely associated with both the four key pathways and inflammatory processes.

\section{Results and Discussion}

3.1. Analysis of Compound-Target Network. The CAS entry, PubChem CID, molecular weight, and content in RDNI of the RDNI compounds are recorded in Table 1. Sixty-seven known targets for 9 compounds and 2 metabolites of RDNI were found in PubChem, BindingDB, and TCMSP (Supplementary Table S1). One hundred and seventeen targets are associated with FDA-approved anti-inflammatory drugs according to DrugBank. Sixty-four targets have 3D structures and can be utilized for molecular docking. Nineteen targets were obtained by molecular docking (Table 2). In total, eighty-four targets were obtained after integration and deduplication. The compound-target network (Figure 1) contains 95 nodes (11 compounds and 84 targets) and 144 edges. Edges in this network reflect the diverse regulations of RDNI for inflammation-related processes.

These compounds and their metabolites have complex interactions with cellular targets. First, caffeic acid (CAA) and its metabolite ferulic acid (FA) have wide influences on inflammatory processes. CAA has 36 targets, and FA has 32 targets, with 20 targets in common. Many targets are related to the regulation of inflammatory process such as arachidonate 5-lipoxygenase (ALOX5, UniProt: P09917), heat shock protein HSP 90-alpha (HSP90AA1, UniProt: P07900), and prostaglandin $\mathrm{G} / \mathrm{H}$ synthase 2 (PTGS2, UniProt: P35354). Second, isochlorogenic acid A (IsoA), isochlorogenic acid B (IsoB), and isochlorogenic acid C (IsoC) have 13, 11, and 9 targets, respectively. Most proteins have explicit relationship with inflammation because they are targets of FDA-approved antiinflammatory drugs. Geniposide (Gen) and its metabolite genipin (Gep) have 12 and 9 targets, respectively, while only one target has affinity with both of them. Third, secoxyloganin (Sec), chlorogenic acid (CGA), cryptochlorogenic acid (4CQA), and neochlorogenic acid (5CQA) have 2, 4, 6, and 6 targets, respectively. They are also important to the therapeutic effect of RDNI as the high content.

Eight targets, namely, thiopurine S-methyl transferase (TPMT, UniProt: P51580), carbonic anhydrase 2 (CA2, UniProt: P00918), amyloid-beta A4 protein (APP, UniProt: P05067), progesterone receptor (PGR, UniProt: P06401), membrane associated phospholipase A2 (PLA2G2A, UniProt: P14555), 5'-AMP-activated protein kinase subunit beta-2 (PRKAB2, UniProt: O43741), aldose reductase (AKR1B1, UniProt: P15121), and PTGS2 would bind with more than 3 compounds of RDNI. Four proteins, namely, 
TABle 2: Targets obtained by molecular docking.

\begin{tabular}{|c|c|c|c|}
\hline Compound & Target & UniProt ID & Binding energy $(\mathrm{kcal} / \mathrm{mol})$ \\
\hline \multirow{5}{*}{ 4CQA } & Progesterone receptor & P06401 & -9.15 \\
\hline & $5^{\prime}$-AMP-activated protein kinase subunit beta- 2 & $\mathrm{O} 43741$ & -9.06 \\
\hline & Thiopurine S-methyltransferase & P51580 & -8.98 \\
\hline & Complement $\mathrm{C} 1 \mathrm{q}$ subcomponent subunit $\mathrm{C}$ & P02747 & -8.51 \\
\hline & Histamine $\mathrm{H} 1$ receptor & P35367 & -8.42 \\
\hline \multirow{5}{*}{ 5CQA } & Progesterone receptor & P06401 & -9.05 \\
\hline & $5^{\prime}$-AMP-activated protein kinase subunit beta- 2 & $\mathrm{O} 43741$ & -8.69 \\
\hline & Phospholipase A2, membrane associated & P14555 & -8.56 \\
\hline & Glutathione S-transferase A2 & P09210 & -8.4 \\
\hline & Retinoic acid receptor alpha & P10276 & -8.38 \\
\hline \multirow{3}{*}{ CGA } & Thiopurine S-methyltransferase & P51580 & -8.74 \\
\hline & Androgen receptor & P10275 & -8.32 \\
\hline & Phospholipase A2, membrane associated & P14555 & -8.28 \\
\hline Gen & Thiopurine S-methyltransferase & P51580 & -8.41 \\
\hline Gep & Corticosteroid 11-beta-dehydrogenase isozyme 1 & P28845 & -8.19 \\
\hline \multirow{9}{*}{ IsoA } & Phospholipase A2, membrane associated & P14555 & -11.82 \\
\hline & $5^{\prime}$-AMP-activated protein kinase subunit beta- 2 & $\mathrm{O} 43741$ & -10.32 \\
\hline & Thiopurine S-methyltransferase & P51580 & -10.24 \\
\hline & Peroxisome proliferator-activated receptor gamma & P37231 & -9.08 \\
\hline & Glucocorticoid receptor & P04150 & -9.02 \\
\hline & Fatty acid-binding protein, intestinal & P12104 & -8.82 \\
\hline & Aldose reductase & P15121 & -8.66 \\
\hline & Histamine $\mathrm{H} 1$ receptor & P35367 & -8.51 \\
\hline & Nitric oxide synthase, endothelial & P29474 & -8.28 \\
\hline \multirow{6}{*}{ IsoB } & Progesterone receptor & P06401 & -10.62 \\
\hline & Thiopurine S-methyltransferase & P51580 & -10.52 \\
\hline & Peroxisome proliferator-activated receptor gamma & P37231 & -9.18 \\
\hline & Prostaglandin reductase 2 & Q8N8N7 & -8.91 \\
\hline & Peroxisome proliferator-activated receptor alpha & Q07869 & -8.89 \\
\hline & Glucocorticoid receptor & P04150 & -8.29 \\
\hline \multirow{8}{*}{ IsoC } & $5^{\prime}$-AMP-activated protein kinase subunit beta- 2 & $\mathrm{O} 43741$ & -10.61 \\
\hline & Progesterone receptor & P06401 & -10.4 \\
\hline & Phospholipase A2, membrane associated & P14555 & -9.97 \\
\hline & Peroxisome proliferator-activated receptor gamma & P37231 & -9.97 \\
\hline & Retinoic acid receptor beta & P10826 & -9.46 \\
\hline & Thiopurine S-methyltransferase & P51580 & -8.89 \\
\hline & Myeloperoxidase & P05164 & -8.54 \\
\hline & Histamine $\mathrm{H} 1$ receptor & P35367 & -8.29 \\
\hline
\end{tabular}

histamine H1 receptor (HRH1, UniProt: P35367), peroxisome proliferator-activated receptor gamma (PPARG, UniProt: P37231), prostaglandin $\mathrm{G} / \mathrm{H}$ synthase 1 (PTGS1, UniProt: P23219), and FAD-linked sulfhydryl oxidase ALR (GFER, UniProt: P55789), can be targeted by 3 compounds of RDNI. These top twelve targets screened by degree centrality have high betweenness centralities (>average value). Seven of them have high closeness centralities (>average value). Other targets can interact with one or two compounds of RDNI. Many of these targets would play significant roles in regulating inflammatory processes.

For example, PTGS2 is the target of CGA, 4CQA, CAA, FA, and Gep. It is the major enzyme responsible for the production of inflammatory prostaglandins and expresses in the inflammatory process only [17]. First, the prostaglandin E2 (PGE2) synthesized by PTGS2 can promote the production of multifarious inflammatory factors, such as interleukin-10 (IL-10, UniProt: P22301) [52], interleukin-8 (IL-8, UniProt: 


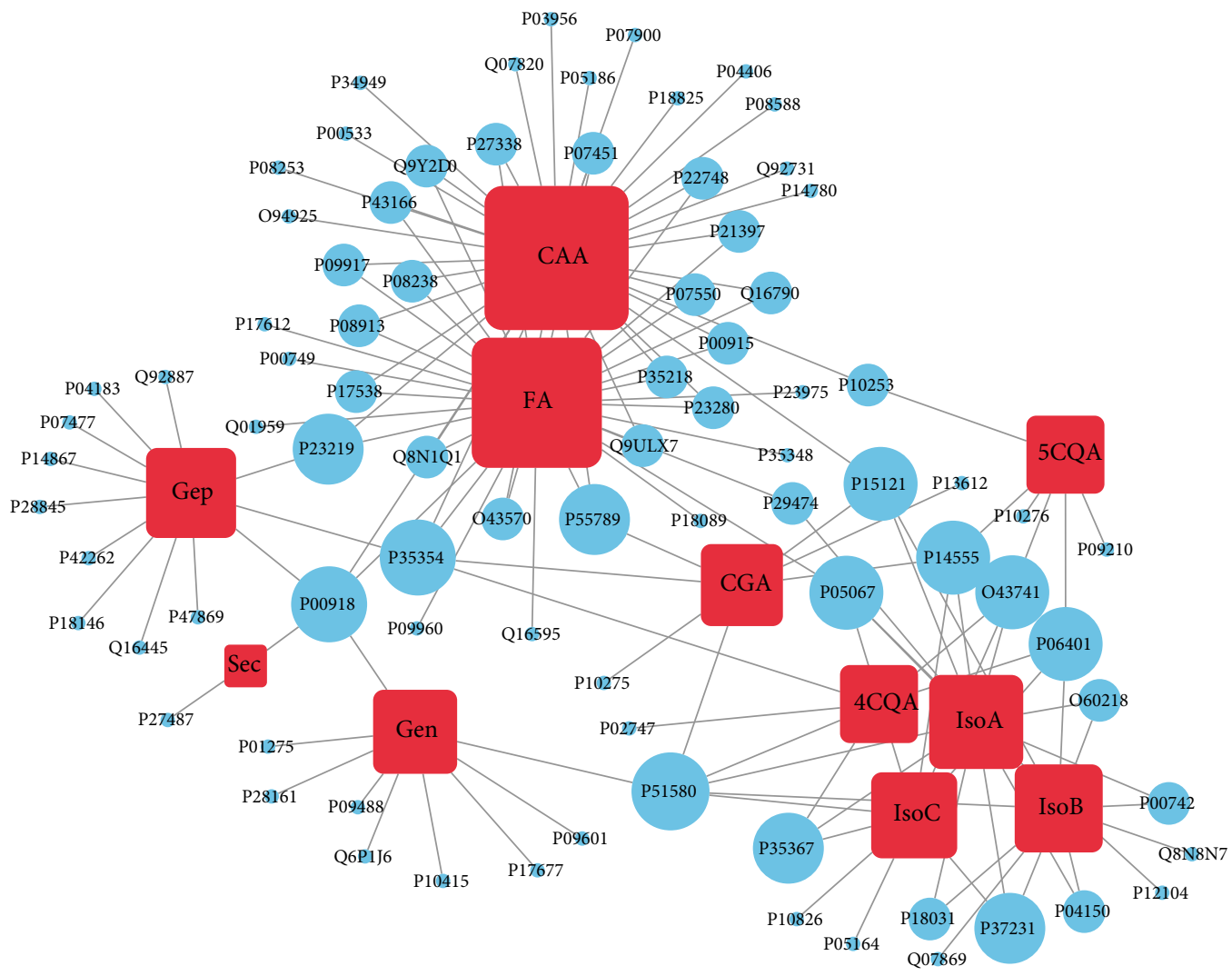

Figure 1: Compound-target network of RDNI. Red squares represent compounds and their metabolites of RDNI, and blue ellipses represent targets. The interaction between compound and target is represented by gray edges. The size of nodes varies with the degree centrality in this network.

P10145), and tumor necrosis factor alpha (TNF $\alpha$, UniProt: P01375) [53]. Second, PGE2 can regulate the production of adenosine $3^{\prime}, 5^{\prime}$-cyclic phosphate (cAMP) and the expressions of inflammatory genes which are regulated by this second messenger [54]. Third, PGE2 is also responsible for some inflammatory symptoms, such as cough [55] and airflow obstruction, because it can regulate the production of mucin in the respiratory tract [56]. Fourth, PTGS2 can influence inflammatory targets such as $72 \mathrm{kDa}$ type IV collagenase (MMP2, UniProt: P08253), matrix metalloproteinase 9 (MMP9, UniProt: P14780), and nitric-oxide synthase, endothelial (eNOS, UniProt: P29474) [57, 58]. Finally, PTGS2 is also the key regulatory factor of differentiation of $\mathrm{T}$ helper cell 17 (Th17) in inflammatory processes [59].

Apoptosis regulator Bcl-2 (Bcl-2, UniProt: P10415) is the target of Gen. After binding with Bcl-2-like protein 1 (Bcl-xl, UniProt: P07817), Bcl-2 can regulate the expression of caspase 1 (CASP1, UniProt: P29466) by inhibiting NLR family protein LRR and PYD domain-containing protein 1 (NALP1, UniProt: Q9C00). CASP1 participates in the dissociation of substrates involved in cell apoptosis and inflammation, and it is capable of catalyzing the maturation and secretion of interleukin-1 beta (IL-1 $\beta$, UniProt: P01584) [60, 61]. NALP1 participates in the process of inflammation by activating the secretion of high mobility group protein B1 (HMGB1, UniProt: P09429). $\mathrm{Bcl}-2$ is also associated with particulate matter-induced pneumonia and allergic airway inflammation by regulating the apoptosis process of inflammatory cells $[62,63]$.

FA and IsoA can bind with eNOS and then regulate the production of nitric oxide (NO), which plays an important role in inflammatory processes [64]. The overproduction of NO participates in inflammatory response by regulating a lot of biological processes, such as the synthesis of iron-nitrite complex, the inhibition of DNA ligase, the promotion of plasma exudation, and edema formation $[65,66]$. The protective effect of eNOS against systemic inflammation is also proved [67].

Peroxisome proliferator-activated receptor alpha (PPARA, UniProt: Q07869) is a member of nuclear hormone receptor superfamily and a target of IsoB. It participates in the regulation of lipid metabolism, adipocyte differentiation, and inflammatory process by activating related transcription factors [68]. Interstitial collagenase (MMP1, UniProt: P03956), MMP2, and MMP9 are both targets of FA and IsoA. MMPs can mediate the pretreatment of TNF $\alpha$ and therefore participate in regulation of inflammatory processes [69]. Induced myeloid leukemia cell differentiation protein Mcl-1 (Mcl-1, UniProt: Q07820) is a target of CAA. It is involved in cell survival, cell apoptosis, and inflammation [70]. Therefore, 


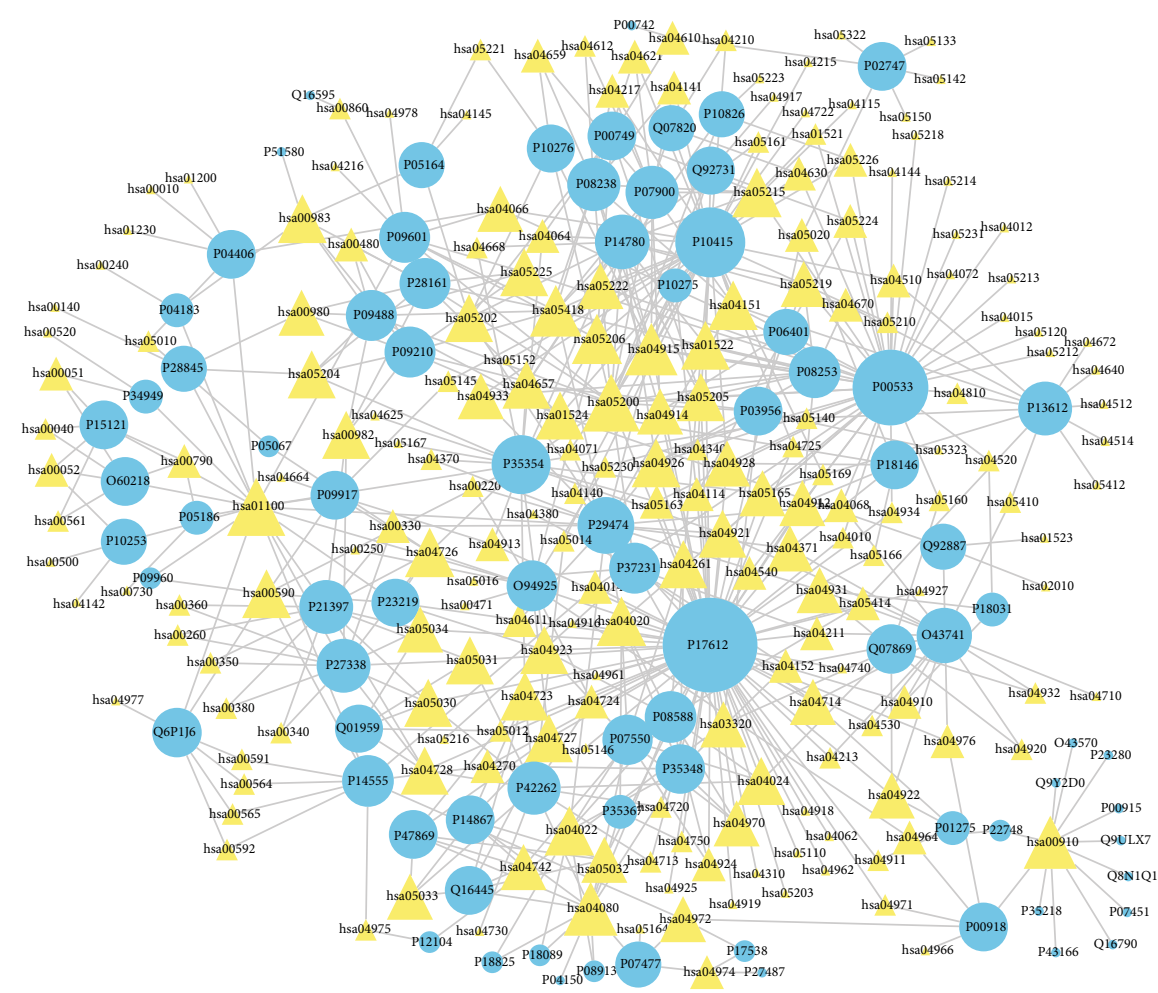

(a)

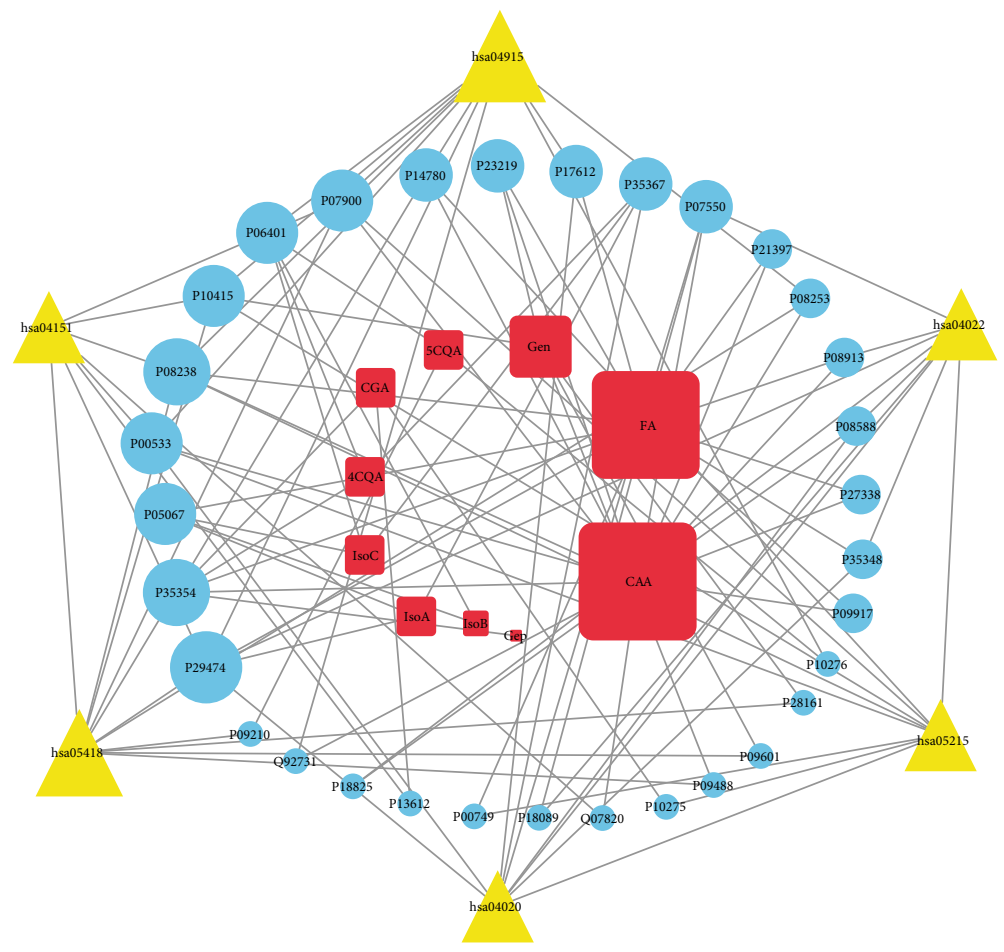

(b)

Figure 2: Target-pathway network (a) and compound-target-pathway network (b). Red squares, blue ellipses, and yellow triangles represent small compounds, targets, and pathways, respectively. Gray edges correspond to the relationships between compounds, targets, and pathways. The size of a node is directly proportional to degree centrality.

compounds of RDNI can regulate the inflammatory process through complex interactions with inflammationassociated targets.
3.2. Analysis of Target-Pathway Network. Eighty targets of RDNI are involved in 201 pathways according to KEGG (Supplementary Table S2). The target-pathway network 


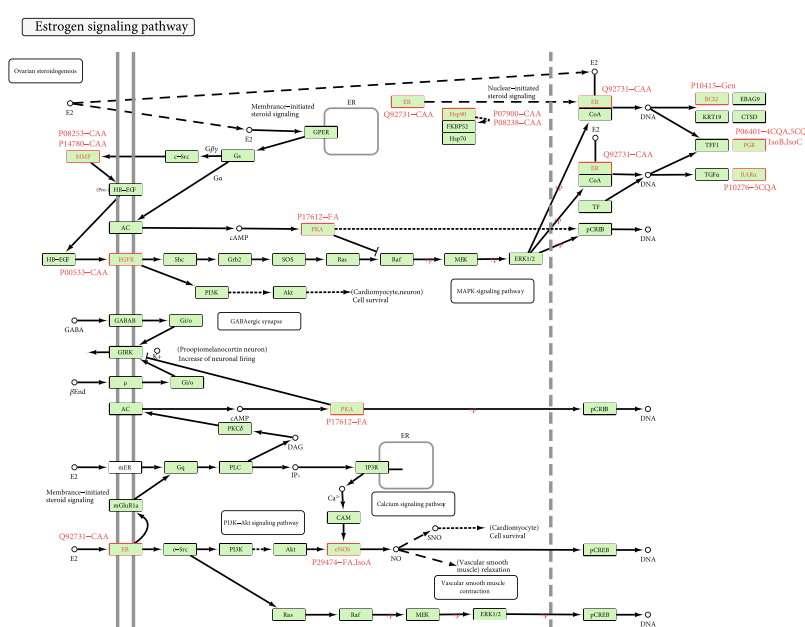

(a)

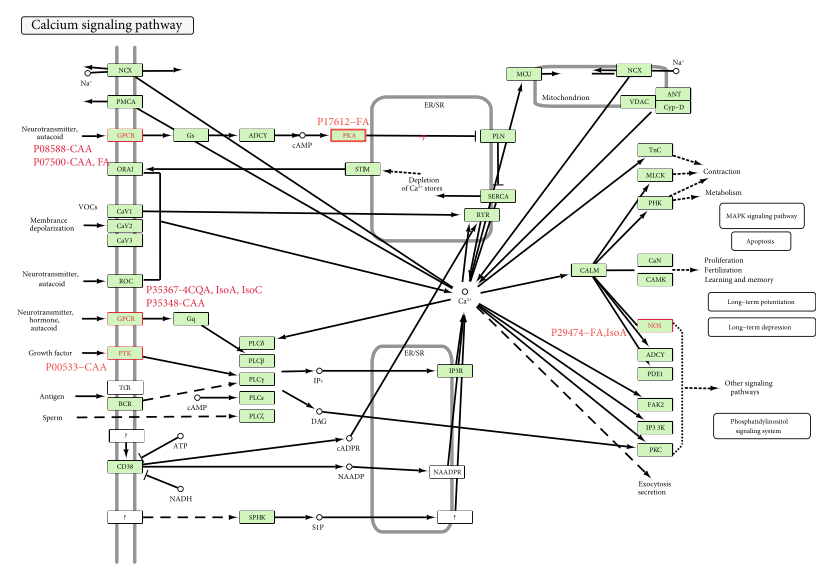

(c)

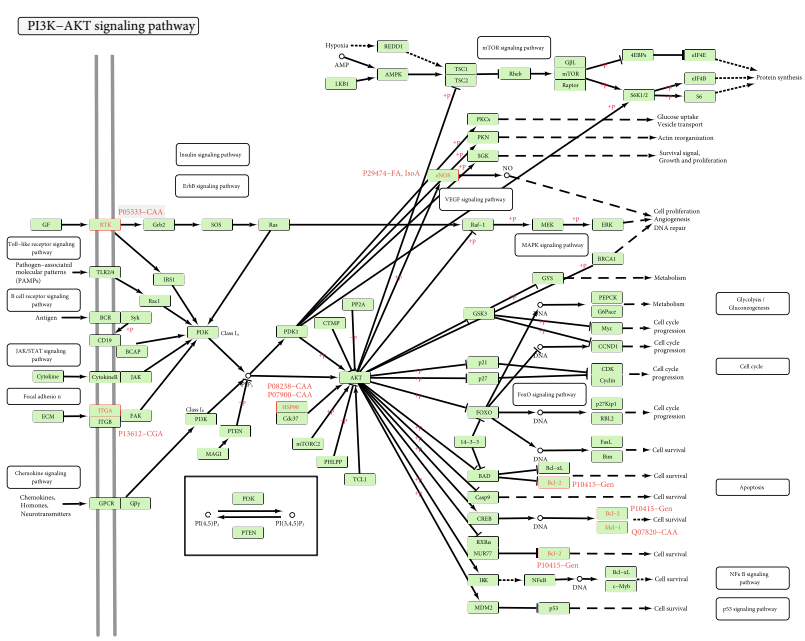

(b)

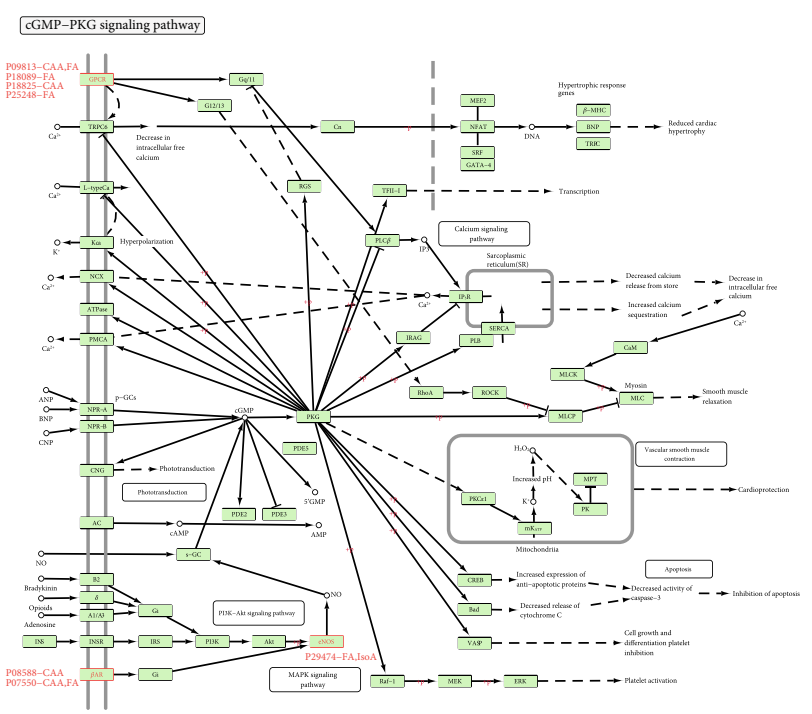

(d)

FIGURe 3: Diagram of the estrogen signaling pathway (a), PI3K-AKT signaling pathway (b), calcium signaling pathway (c), and cGMP-PKG signaling pathway (d). Names of each pathway are labeled in the upper left corner. The origin pathway images are downloaded from KEGG. The targets of RDNI are marked in red. UniProt ID of a specific target and the compound(s) binding with it are labeled nearby. Permission is granted by Kanehisa Laboratories to use these KEGG pathway map images.

(Figure 2(a)) contains 281 nodes (80 targets and 201 pathways) and 577 edges. The TPN shows the complexity and diversity of regulatory effects of RDNI on human biological processes. Twelve pathways would have close relationship with inflammation since they have large degree centrality $(>6)$. Six pathways are excluded because they do not represent a specific biological process or do not have high closeness centralities, namely, metabolic pathways, pathways in cancer, neuroactive ligand-receptor interaction, nitrogen metabolism, serotonergic synapse, and microRNAs in cancer. The rest 6 pathways constitute the compound-target-pathway network (Figure 2(b)) by linking 32 targets and 10 compounds. The CTPN contains 48 nodes and 102 edges. Figures 2(a) and 2(b) reflect the multiple regulatory functions of RDNI through different pathways. Four of them were found to have high correlation with the regulation of inflammatory response, namely, estrogen signaling pathway (hsa04915), PI3K-AKT signaling pathway (hsa04151), cGMP-PKG signaling pathway (hsa04022), and calcium signaling pathway (hsa04020).

The estrogen signaling pathway (Figure 3(a)) contains the most targets of RDNI than other pathways. It regulates many physiological processes such as reproduction, cardiovascular protection, cellular homeostasis, and inflammatory metabolic process [71-73]. Eight compounds (CAA, FA, 4CQA, 5CQA, IsoA, IsoB, IsoC, and Gen) can interact with eleven targets (P00533, P06401, P07900, P08238, P08253, P10276, P10415, P14780, P17612, P29474, and Q92731) in this pathway. First, CAA can regulate the estrogen signaling pathway by binding with HSP90 and modulate estrogen receptor beta (ESR2, UniProt: Q92731). Second, HSP90 is involved in the formation process of the complex of estrogen receptor (ER) and nuclear receptor coactivator (CoA). The 


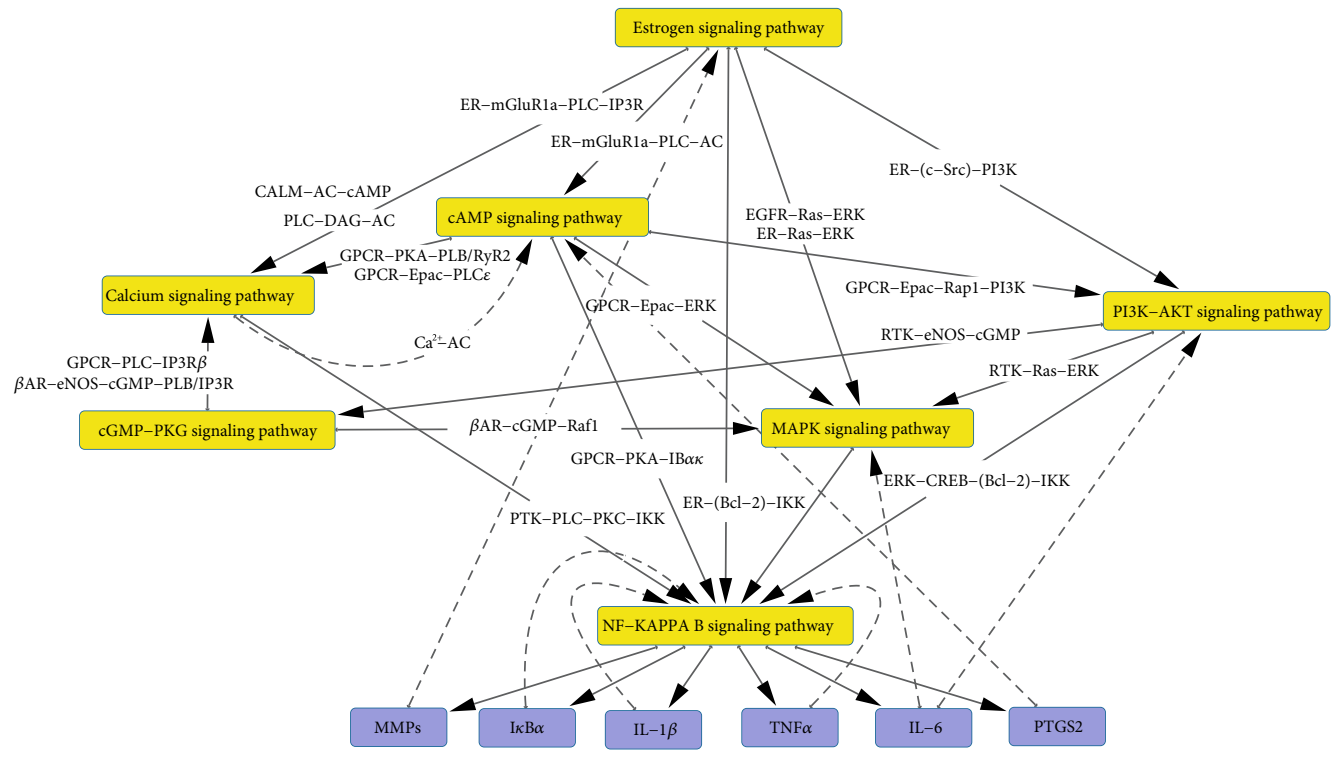

FIGURE 4: Cross-talks between seven primary regulatory pathways of RDNI for inflammation. Gray edge represents that the pathway in front of the arrow is regulated by the upstream pathway. The connection between two pathways is marked on the edge. The feedback regulations are marked as dashed lines.

ER-CoA complex can activate the expression of ERdependent genes, which participate in the regulation of cell apoptosis and inflammation, such as Bcl-2, PGR, and retinoic acid receptor alpha (RARA, UniProt: P10276). Third, MMP2, MMP9, ER, and epidermal growth factor receptor (EGFR, UniProt: P00533) can regulate second messengers that play important roles in other inflammation-related pathways, such as cAMP, $\mathrm{Ca}^{2+}$, and phosphatidylinositol-3,4,5trisphosphate $\left(\mathrm{PIP}_{3}\right)$. The compounds of RDNI can regulate the inflammatory process by regulating the estrogen signaling pathway in many ways.

The PI3K-AKT signaling pathway (Figure $3(\mathrm{~b})$ ) is responsible for multiple fundamental cellular functions by the phosphorylation of serine/threonine kinase (AKT) [7476]. It can also regulate inflammatory processes in many approaches [77]. Five RDNI compounds (CAA, FA, CGA, IsoA, and Gen) have influences on seven targets of this pathway (P00533, P07900, P08238, P10415, P13612, P29474, and Q07820). CAA can inhibit EGFR and then regulate the production of $\mathrm{PIP}_{3}$. PIP 3 , as well as the complex of HSP90 and Hsp90 cochaperone Cdc37 (UniProt: Q16543), can activate AKT $[78,79]$. Activation of AKT leads to phosphorylation of downstream targets which are associated with the inflammatory process. For example, the phosphorylation of inhibitor of nuclear factor kappa-B kinase (IKK) promotes the dissociation of the complex of NF-kappa-B inhibitor alpha $(\mathrm{I} \kappa \mathrm{B} \alpha)$ and nuclear factor kappa-B (NF- $\kappa \mathrm{B})$, and then, NF$\kappa \mathrm{B}$ is released [80]. The phosphorylation of eNOS has an impact on the production of NO in the body. The phosphorylation of cyclic AMP-responsive element-binding protein (CREB) activates the expressions of Bcl-2 and Mcl-1. The phosphorylation of $\mathrm{Bcl} 2$-associated agonist of cell death (BAD, UniProt: Q92934) inhibits the expression of Bcl-2 and Bcl-xl. The phosphorylation of the complex of retinoic acid receptor RXR-alpha (RXRA, UniProt: P19793) and nuclear receptor subfamily 4 group A member 1 (NUR77) inhibits the expression of Bcl-2. Compounds of RDNI, such as FA, IsoA, and Gen, can also regulate these inflammation-related targets directly.

The calcium signaling pathway (Figure 3(c)) maintains the equilibrium of calcium concentration in the body and therefore mediates signal transduction in cellular and physiological processes $[81,82]$. Five compounds of RDNI (CAA, FA, IsoA, IsoC, and 4CQA) would bind with seven targets of this pathway (P00533, P07550, P08588, P17612, P29474, P35348, and P35367). The impact of these compounds on the calcium signaling pathway is reflected in the regulation of phospholipase C (PLC). First, CAA regulates PLC $\gamma$ by inhibiting EGFR in the calcium signaling pathway. Second, HRH1, a subtype of G-protein coupled receptor (GPCR), is the target of FA, IsoA, 4CQA, and IsoC and activates PLC $\beta$. Third, cAMP is the activator of PLCE and also regulates PLC $\delta$ by regulating $\mathrm{Ca}^{2+}$ concentration. PLC produces $\mathrm{D}$ myo-inositol 1,4,5-trisphosphate (IP3) and diacylglycerol (DAG); then, IP3 and DAG activate protein kinase C (PKC). PKC can regulate lipopolysaccharide-induced macrophage functions involved in inflammation [83]. It can also participate in inflammatory response by regulating NF- $\kappa \mathrm{B}$ induced gene expression through the IL- $1 \alpha$-dependent induction of $\mathrm{I} \kappa \mathrm{B} \alpha[84]$.

The cGMP-PKG signaling pathway (Figure $3(\mathrm{~d})$ ) regulates a broad array of physiologic processes, such as vascular smooth muscle contraction, cell apoptosis, and inflammation [85]. The regulatory function is implemented through the phosphorylation function of cGMP-dependent protein kinase (PKG), a downstream protein of $3^{\prime}, 5^{\prime}$-cyclic GMP 
(cGMP) [86, 87]. Three RDNI compounds (CAA, FA, and IsoA) can interact with seven targets of this pathway (P07550, P08588, P08913, P18089, P18825, P29474, and P35348). CAA and FA can interact with alpha adrenergic receptors ( $\alpha$ ARs, P08913, P18089, P18825, and P35348) and beta adrenergic receptors ( $\beta$ ARs, P07550, and P08588). $\alpha$ ARs activate guanine nucleotide-binding protein subunit alpha-11 (GNA11, UniProt: P29992) and guanine nucleotide-binding protein $\mathrm{G}(\mathrm{q})$ subunit alpha (GNAQ, UniProt: P50148). GAN11 and GANQ can further mediate the generation of $\mathrm{Ca}^{2+}$ by activating IP3R. $\beta$ ARs would activate guanine nucleotide-binding protein $\mathrm{G}(\mathrm{i})$ subunit alpha (Gi). Gi can conduct the stimulus signal to eNOS and then mediate the generation of $\mathrm{NO}$, while $\mathrm{FA}$ and IsoA can bind with eNOS directly. NO regulates the generation of cGMP by activating s-GC. cGMP can activate PKG which regulates multitudinous targets, including inflammation-related targets (CREB, BAD) and targets responsible for $\mathrm{Ca}^{2+}$ concentrate in the body, such as PLC $\beta$, protein MRVI1 (UniProt: Q9Y6F6), and cardiac phospholamban (PLB, UniProt: P26678).

The other two pathways are fluid shear stress and atherosclerosis pathway (hsa05418) and prostate cancer pathway (hsa05215). The fluid shear stress and atherosclerosis pathway regulates the progress of atherosclerosis and correlates with the activation of proinflammatory gene expression as well as early atherogenic inflammation [88, 89]. RDNI can also regulate the prostate cancer pathway by interacting with 7 targets. This pathway has connection with both inflammation-related targets and prostate cancer, indicating that RDNI may have potential therapeutic effect against prostate cancer [90]. Degree centralities, betweenness centralities, and closeness centralities of other pathways in Figures 2(a) and 2(b) are generally lower, but they are also important in the inflammatory process, such as IL-17 signaling pathway, NF- $\kappa \mathrm{B}$ signaling pathway, and arachidonic acid metabolism pathway. It is worth mentioning that these pathways are closely relevant to the former four pathways.

3.3. Cross-Talks among Inflammation-Related Pathways. Cross-talks among pathways are common in the regulation of biological processes. They are normally connected by key targets or common upstream/downstream pathways. The integration and correlation analysis of these pathways can help understand the MOA of TCM thoroughly and comprehensively. We analyzed the cross-talks within four key inflammation-related pathways regulated by RDNI, namely, estrogen signaling pathway, PI3K-AKT signaling pathway, cGMP-PKG signaling pathway, and calcium signaling pathway. Another three pathways were introduced to bridge the gaps among these key pathways, namely, cAMP signaling pathway (hsa04024), MAPK signaling pathway (hsa04010), and NF- $\kappa$ B signaling pathway (hsa04064). The cross-talk network (Figure 4) shows that each pathway is directly related to the NF- $\kappa \mathrm{B}$ signaling pathway. The expression products of NF- $\kappa$ B, such as IL- $1 \beta$ [91], TNF $\alpha$ [92], PTGS2, interleukin 6 (IL-6) [93], MMPs, and Bcl-2, play significant roles in the inflammatory process. Associations with NF- $\kappa \mathrm{B}$ signaling pathways suggest the importance of other pathways in the inflammatory process. The estrogen signaling pathway is located in the upstream of the whole regulatory network, and therefore, CAA can link almost all inflammatory processes in the downstream pathway by modulating ER. Meanwhile, ER can regulate the expression of BCL2 gene and then the expression of NF- $\kappa \mathrm{B}$ as well as inflammatory factors succinctly. The cAMP signaling pathway is the direct upstream of the PI3K-AKT signaling pathway, calcium signaling pathway, and NF- $\kappa$ B signaling pathway; hence, CAA and its derivative FA can influence the downstream inflammatory process by regulating the formation of the cAMP.

The MAPK signaling pathway connects the estrogen signaling pathway, PI3K-AKT signaling pathway, cGMP-PKG signaling pathway, and cAMP signaling pathway to the expression of NF- $\kappa \mathrm{B}$ through the Ras pathway. In the calcium signaling pathway, the generation of $\mathrm{Ca}^{2+}$ is regulated by cAMP through multiple approaches. $\mathrm{Ca}^{2+}$ can regulate the expression of cAMP in the form of feedback by $\mathrm{Ca}^{2+}$ dependent adenylate cyclase (AC) and PKC $\theta$. The generation of cGMP is regulated by $\mathrm{NO}$ whose concentrate is regulated by eNOS, a downstream enzyme of AKT. It can also interact with regulatory targets of $\mathrm{Ca}^{2+}$ just like cAMP. The expression product of NF- $\kappa \mathrm{B}$ such as IL- $1 \beta, \mathrm{TNF} \alpha$, and $\mathrm{I} \kappa \mathrm{B} \alpha$ regulates both the expression of IKK and the dissociation of the $\mathrm{I} \kappa \mathrm{B} \alpha-\mathrm{NF}-\kappa \mathrm{B}$ complex. The generation of IL-6, PTGS2, and MMPs also has feedback regulation on upstream pathways. These feedback regulation and the mediation of the MAPK signaling pathway increase the complexity and robustness of the regulatory effects of RDNI on the inflammatory process.

\section{Conclusions}

In this work, the anti-inflammatory mechanism of RDNI was explored by network pharmacological methods. Eighty-four targets of RDNI were collected by data mining and molecular docking to construct a compound-target network. Key targets (Bcl-2, eNOS, PTGS2, PPARA, and MMPs) were found to be responsible for regulating the inflammatory process by RDNI compounds and metabolites. Two hundred and one pathways were found to be connected with RDNI targets. Four key pathways, namely, estrogen signaling pathway, PI3K-AKT signaling pathway, cGMP-PKG signaling pathway, and calcium signaling pathway, would play important roles. The cross-talks among four key pathways and another three related pathways were further identified. Results demonstrate that RDNI, an injection formed by multiple ingredients, can interact with multifarious inflammation-related targets. The interactions make RDNI capable of regulating multiple biological processes and treat inflammation at the systems level. Moreover, TCM is a complicated drug system; thus, complex interactions between multicomponents and multitargets make it possible to regulate multipathways and biological processes. Although the conclusions obtained in this research require to be verified by further experiments, network pharmacology provides a promising approach to investigate the MOA of TCM. 


\section{Data Availability}

The data used to support the findings of this study are included within the supplementary information files.

\section{Conflicts of Interest}

The authors declare that there is no conflict of interest regarding the publication of this paper.

\section{Acknowledgments}

The calculations were performed on a high-performance computer cluster of Guangdong Provincial Hospital of Traditional Chinese Medicine. This work was supported by the start-up support for scientific research of Xinglin Young Scholar in Guangzhou University of Chinese Medicine (A1AFD018161Z04), National Natural Science Foundation of China (81904198 and 81804111), and Guangdong Provincial Hospital of Traditional Chinese Medicine Science and Technology Research Program (YN2016MJ06).

\section{Supplementary Materials}

Supplementary Table S1: targets gathered in PubChem, BindingDB, and TCMSP. Supplementary Table S2: network topological parameters of CTN. Supplementary Table S3: target-pathway relationships of potential targets of RDNI. Supplementary Table S4: network topological parameters of TPN. Supplementary Figure S1: HPLC spectra of RDNI at $237 \mathrm{~nm}$ (a) and $324 \mathrm{~nm}$ (b). (Supplementary Materials)

\section{References}

[1] C. N. Jenne and P. Kubes, "Platelets in inflammation and infection," Platelets, vol. 26, no. 4, pp. 286-292, 2015.

[2] A. Patel, "Review: the role of inflammation in depression," Psychiatr Danub, vol. 25, Supplement 2, pp. S216-S223, 2013.

[3] L. F. Shirazi, J. Bissett, F. Romeo, and J. L. Mehta, "Role of inflammation in heart failure," Curr Atheroscler Rep, vol. 19, no. 6, p. $27,2017$.

[4] K. Nishida and K. Otsu, "Inflammation and metabolic cardiomyopathy," Cardiovascular Research, vol. 113, no. 4, pp. 389398, 2017.

[5] C. Nathan, "Points of control in inflammation," Nature, vol. 420, no. 6917, pp. 846-852, 2002.

[6] K. J. Tracey, "The inflammatory reflex," Nature, vol. 420, no. 6917, pp. 853-859, 2002.

[7] R. Medzhitov, "Origin and physiological roles of inflammation," Nature, vol. 454, no. 7203, pp. 428-435, 2008.

[8] D. Laveti, M. Kumar, R. Hemalatha et al., "Anti-inflammatory treatments for chronic diseases: a review," Inflammation \& Allergy Drug Targets, vol. 12, no. 5, pp. 349-361, 2013.

[9] G. Egger and J. Dixon, "Beyond obesity and lifestyle: a review of 21st century chronic disease determinants," BioMed Research International, vol. 2014, Article ID 731685, pp. 1-12, 2014.

[10] L. Cicchitti, M. Martelli, and F. Cerritelli, "Chronic inflammatory disease and osteopathy: a systematic review," PLoS One, vol. 10, no. 3, p. e0121327, 2015.
[11] S. Bacchi, P. Palumbo, A. Sponta, and M. F. Coppolino, "Clinical pharmacology of non-steroidal anti-inflammatory drugs: a review," Anti-Inflammatory \& Anti-Allergy Agents in Medicinal Chemistry, vol. 11, no. 1, pp. 52-64, 2012.

[12] S. A. Klinge and G. A. Sawyer, "Effectiveness and safety of topical versus oral nonsteroidal anti-inflammatory drugs: a comprehensive review," The Physician and Sportsmedicine, vol. 41, no. 2, pp. 64-74, 2015.

[13] A. K. Dhingra, B. Chopra, R. Dass, and S. K. Mittal, "An update on anti-inflammatory compounds: a review," AntiInflammatory \& Anti-Allergy Agents in Medicinal Chemistry, vol. 14, no. 2, pp. 81-97, 2015.

[14] S. Ahmad, A. Bhanji, S. Pal, and M. Karim, "Irreversible sensorineural hearing loss: an unusual side effect of non-steroidal anti-inflammatory drugs," International Journal of Clinical Pharmacology and Therapeutics, vol. 48, no. 8, pp. 514-516, 2010.

[15] P. R. Yap and K. L. Goh, "Non-steroidal anti-inflammatory drugs (NSAIDs) induced dyspepsia," Current Pharmaceutical Design, vol. 21, no. 35, pp. 5073-5081, 2015.

[16] E. Petzold, W. Richter, and H. J. Karschunke, "Side effects of nonsteroidal and steroidal anti-inflammatory drugs on cartilage metabolism-facts from research and practice," Zeitschrift für Alternsforschung, vol. 40, no. 4, pp. 253-259, 1985.

[17] X. Zhang, J. Gu, L. Cao et al., "Insights into the inhibition and mechanism of compounds against LPS-induced PGE2production: a pathway network-based approach and molecular dynamics simulations," Integr. Biol, vol. 6, no. 12, pp. 1162$1169,2014$.

[18] K. Wang, D. Zhang, Y. Liu et al., "Traditional Chinese medicine formula $\mathrm{Bi}-\mathrm{Qi}$ capsule alleviates rheumatoid arthritisinduced inflammation, synovial hyperplasia, and cartilage destruction in rats," Arthritis Res Ther, vol. 20, no. 1, p. 43, 2018.

[19] N. Gu, Y. Tian, Z. Di, C. Han, H. Lei, and G. Zhang, "Shuanghuanglian injection downregulates nuclear factor-kappa $B$ expression in mice with viral encephalitis," Neural Regeneration Research, vol. 7, no. 33, pp. 2592-2599, 2012.

[20] Y. M. Ma, X. Z. Zhang, Z. Z. Su et al., "Insight into the molecular mechanism of a herbal injection by integrating network pharmacology and in vitro," Journal of Ethnopharmacology, vol. 173, pp. 91-99, 2015.

[21] Y. J. Li, Y. Guo, Q. Yang et al., "Flavonoids casticin and chrysosplenol D from Artemisia annua L. inhibit inflammation in vitro and in vivo," Toxicology and Applied Pharmacology, vol. 286, no. 3, pp. 151-158, 2015.

[22] X. Zhang, J. Gu, L. Cao et al., "Network pharmacology study on the mechanism of traditional Chinese medicine for upper respiratory tract infection," Molecular BioSystems, vol. 10, no. 10, pp. 2517-2525, 2014.

[23] A. L. Hopkins, "Network pharmacology: the next paradigm in drug discovery," Nature Chemical Biology, vol. 4, no. 11, pp. 682-690, 2008.

[24] L. I. Furlong, "Human diseases through the lens of network biology," Trends in Genetics, vol. 29, no. 3, pp. 150-159, 2013.

[25] A. D. Boran and R. Iyengar, "Systems approaches to polypharmacology and drug discovery," Current Opinion in Drug Discovery \& Development, vol. 13, no. 3, pp. 297-309, 2010.

[26] T. Chen, J. Gu, X. Zhang et al., "System-level study on synergism and antagonism of active ingredients in traditional 
Chinese medicine by using molecular imprinting technology," Scientific Reports, vol. 4, no. 1, p. 7159, 2015.

[27] F. Luo, J. Gu, X. Zhang et al., "Multiscale modeling of druginduced effects of ReDuNing injection on human disease: from drug molecules to clinical symptoms of disease," Scientific Reports, vol. 5, no. 1, p. 10064, 2015.

[28] Y. Liu, W. Mu, W. Xiao et al., "Efficacy and safety of Re-DuNing injection in the treatment of seasonal influenza: results from a randomized, double-blinded, multicenter, oseltamivircontrolled trial," Oncotarget, vol. 8, no. 33, pp. 55176-55186, 2017.

[29] X. Shang, H. Pan, M. Li, X. Miao, and H. Ding, "Lonicera japonica Thunb.: ethnopharmacology, phytochemistry and pharmacology of an important traditional Chinese medicine," Journal of Ethnopharmacology, vol. 138, no. 1, pp. 1-21, 2011.

[30] T. Li, H. Chen, N. Wei et al., "Anti-inflammatory and immunomodulatory mechanisms of artemisinin on contact hypersensitivity," International Immunopharmacology, vol. 12, no. 1, pp. 144-150, 2012.

[31] Y. Deng, M. Guan, X. Xie et al., "Geniposide inhibits airway inflammation and hyperresponsiveness in a mouse model of asthma," International Immunopharmacology, vol. 17, no. 3, pp. 561-567, 2013.

[32] Y. J. Li, Z. Z. Wang, Y. A. Bi et al., "Direct analysis in real time ionization/quadrupole time-of-flight tandem mass spectrometry for rapid identification of iridoid glycosides and caffeoylquinic acids in Re Du Ning injections," Analytical Methods, vol. 5, no. 24, pp. 7081-7089, 2013.

[33] Y. J. Li, Z. Z. Wang, Y. A. Bi et al., "The evaluation and implementation of direct analysis in real time quadrupole time-offlight tandem mass spectrometry for characterization and quantification of geniposide in Re Du Ning injections," Rapid Communications in Mass Spectrometry, vol. 26, no. 11, pp. 1377-1384, 2012.

[34] H. Li, Studies on Pharmacodynamic Material Basis of Redlining Injection, Nanjing University of Chinese Medicine, 2013.

[35] D. Kang, T. Geng, Y. Lian et al., "Direct inhibition of Re Du Ning Injection and its active compounds on human liver cytochrome P450 enzymes by a cocktail method," Biomed Chromatogr, vol. 31, no. 7, 2017.

[36] S. Zhang, Y. J. Li, C. X. Zhang et al., "Research on the change of chemical composition in productive process of Re Du Ning injection by HPLC/Q-TOF MS," Biomedical Chromatography, vol. 30, no. 2, pp. 131-141, 2016.

[37] L. Li, Z. Wang, Y. Peng et al., "Screening and identification of multi-components in Re Du Ning injections using LC/TOFMS coupled with UV-irradiation," Journal of Chromatographic Science, vol. 53, no. 5, pp. 778-786, 2015.

[38] H. Li, Y. Yu, Z. Wang et al., "Chemical profiling of Re-DuNing injection by ultra-performance liquid chromatography coupled with electrospray ionization tandem quadrupole time-of-flight mass spectrometry through the screening of diagnostic Ions in MS(E) mode," PLoS One, vol. 10, no. 4, p. e0121031, 2015.

[39] L. I. Mennen, D. Sapinho, H. Ito et al., "Urinary flavonoids and phenolic acids as biomarkers of intake for polyphenol-rich foods," The British Journal of Nutrition, vol. 96, no. 1, pp. 191-198, 2006.

[40] M. Y. Moridani, H. Scobie, and P. J. O'Brien, "Metabolism of caffeic acid by isolated rat hepatocytes and subcellular fractions," Toxicology Letters, vol. 133, no. 2-3, pp. 141151, 2002.

[41] T. Khanal, H. G. Kim, J. H. Choi et al., "Biotransformation of geniposide by human intestinal microflora on cytotoxicity against HepG2 cells," Toxicology Letters, vol. 209, no. 3, pp. 246-254, 2012.

[42] S. Kim, P. A. Thiessen, E. E. Bolton et al., "PubChem substance and compound databases," Nucleic Acids Research, vol. 44, no. D1, pp. D1202-D1213, 2016.

[43] J. Ru, P. Li, J. Wang et al., "TCMSP: a database of systems pharmacology for drug discovery from herbal medicines," Journal of Cheminformatics, vol. 6, no. 1, p. 13, 2014.

[44] X. Chen, Y. Lin, and M. K. Gilson, "The binding database: overview and user's guide," Biopolymers, vol. 61, no. 2, pp. 127-141, 2001.

[45] X. Chen, Y. Lin, M. Liu, and M. K. Gilson, "The binding database: data management and interface design," Bioinformatics, vol. 18, no. 1, pp. 130-139, 2002.

[46] D. S. Wishart, Y. D. Feunang, A. C. Guo et al., "DrugBank 5.0: a major update to the DrugBank database for 2018," Nucleic Acids Research, vol. 46, no. D1, pp. D1074-D1082, 2018.

[47] G. M. Morris, R. Huey, W. Lindstrom et al., “AutoDock4 and AutoDockTools4: automated docking with selective receptor flexibility," Journal of Computational Chemistry, vol. 30, no. 16, pp. 2785-2791, 2009.

[48] P. Shannon, A. Markiel, O. Ozier et al., "Cytoscape: a software environment for integrated models of biomolecular interaction networks," Genome Research, vol. 13, no. 11, pp. 24982504, 2003.

[49] Y. Assenov, F. Ramirez, S. E. Schelhorn, T. Lengauer, and M. Albrecht, "Computing topological parameters of biological networks," Bioinformatics, vol. 24, no. 2, pp. 282-284, 2008.

[50] T. UniProt Consortium, "UniProt: the universal protein knowledgebase," Nucleic Acids Res, vol. 46, no. 5, p. 2699, 2018.

[51] M. Kanehisa, M. Furumichi, M. Tanabe, Y. Sato, and K. Morishima, "KEGG: new perspectives on genomes, pathways, diseases and drugs," Nucleic Acids Research, vol. 45, no. D1, pp. D353-d361, 2017.

[52] K. F. MacKenzie, K. Clark, S. Naqvi et al., "PGE(2) induces macrophage IL-10 production and a regulatory-like phenotype via a protein kinase A-SIK-CRTC3 pathway," Journal of Immunology, vol. 190, no. 2, pp. 565-577, 2013.

[53] F. Neuschafer-Rube, A. Pathe-Neuschafer-Rube, S. Hippenstiel, and G. P. Puschel, "PGE2 enhanced TNFalpha-mediated IL-8 induction in monocytic cell lines and PBMC," Cytokine, vol. 113, pp. 105-116, 2018.

[54] C. K. Billington, O. O. Ojo, R. B. Penn, and S. Ito, "cAMP regulation of airway smooth muscle function," Pulmonary Pharmacology \& Therapeutics, vol. 26, no. 1, pp. 112-120, 2013.

[55] Y. Kawakami, K. Uchiyama, T. Irie, and M. Murao, "Evaluation of aerosols of prostaglandins E1 and E2 as bronchodilators," European Journal of Clinical Pharmacology, vol. 6, no. 2, pp. 127-132, 1973.

[56] Z. Marom, J. H. Shelhamer, and M. Kaliner, "Effects of arachidonic acid, monohydroxyeicosatetraenoic acid and prostaglandins on the release of mucous glycoproteins from human airways in vitro," The Journal of Clinical Investigation, vol. 67, no. 6, pp. 1695-1702, 1981.

[57] W. T. Loo, M. Wang, L. J. Jin, M. N. Cheung, and G. R. Li, "Association of matrix metalloproteinase (MMP-1, MMP-3 
and MMP-9) and cyclooxygenase-2 gene polymorphisms and their proteins with chronic periodontitis," Archives of Oral Biology, vol. 56, no. 10, pp. 1081-1090, 2011.

[58] J. B. Weinberg, "Nitric oxide synthase 2 and cyclooxygenase 2 interactions in inflammation," Immunologic Research, vol. 22, no. 2-3, pp. 319-342, 2000.

[59] H. Li, J. A. Bradbury, R. T. Dackor et al., "Cyclooxygenase-2 regulates Th17 cell differentiation during allergic lung inflammation," American Journal of Respiratory and Critical Care Medicine, vol. 184, no. 1, pp. 37-49, 2011.

[60] J. M. Bruey, N. Bruey-Sedano, F. Luciano et al., "Bcl-2 and Bcl-XL regulate proinflammatory caspase-1 activation by interaction with NALP1," Cell, vol. 129, no. 1, pp. 45-56, 2007.

[61] J. N. Finger, J. D. Lich, L. C. Dare et al., “Autolytic proteolysis within the function to find domain (FIIND) is required for NLRP1 inflammasome activity," The Journal of Biological Chemistry, vol. 287, no. 30, pp. 25030-25037, 2012.

[62] B. P. Tian, L. X. Xia, Z. Q. Bao et al., "Bcl-2 inhibitors reduce steroid-insensitive airway inflammation," The Journal of Allergy and Clinical Immunology, vol. 140, no. 2, pp. 418430, 2017.

[63] X. Geng, X. Wang, M. Luo et al., "Induction of neutrophil apoptosis by a Bcl-2 inhibitor reduces particulate matter-induced lung inflammation," Aging (Albany NY), vol. 10, no. 6, pp. 1415-1423, 2018.

[64] T. J. Guzik, R. Korbut, and T. Adamek-Guzik, "Nitric oxide and superoxide in inflammation and immune regulation," Journal of Physiology and Pharmacology, vol. 54, no. 4, pp. 469-487, 2003.

[65] D. A. Wink and J. B. Mitchell, "Chemical biology of nitric oxide: insights into regulatory, cytotoxic, and cytoprotective mechanisms of nitric oxide," Free Radical Biology \& Medicine, vol. 25, no. 4-5, pp. 434-456, 1998.

[66] T. Akaike, M. Suga, and H. Maeda, "Free radicals in viral pathogenesis: molecular mechanisms involving superoxide and NO," Proceedings of the Society for Experimental Biology and Medicine, vol. 217, no. 1, pp. 64-73, 1998.

[67] M. Bougaki, R. J. Searles, K. Kida, J. Yu, E. S. Buys, and F. Ichinose, "Nos3 protects against systemic inflammation and myocardial dysfunction in murine polymicrobial sepsis," Shock, vol. 34, no. 3, pp. 281-290, 2010.

[68] A. Gorla-Bajszczak, C. Juge-Aubry, A. Pernin, A. G. Burger, and C. A. Meier, "Conserved amino acids in the ligandbinding and $\tau \mathrm{i}$ domains of the peroxisome proliferatoractivated receptor $\alpha$ are necessary for heterodimerization with RXR," Molecular and Cellular Endocrinology, vol. 147, no. 1-2, pp. 37-47, 1999.

[69] A. J. Gearing, P. Beckett, M. Christodoulou et al., "Matrix metalloproteinases and processing of pro-TNF- $\alpha$," Journal of Leukocyte Biology, vol. 57, no. 5, pp. 774-777, 1995.

[70] U. Maurer, C. Charvet, A. S. Wagman, E. Dejardin, and D. R. Green, "Glycogen synthase kinase-3 regulates mitochondrial outer membrane permeabilization and apoptosis by destabilization of MCL-1," Molecular Cell, vol. 21, no. 6, pp. 749-760, 2006.

[71] G. R. do Nascimento, Y. Barros, A. Wells, and R. Khalil, "Research into specific modulators of vascular sex hormone receptors in the management of postmenopausal cardiovascular disease," Current Hypertension Reviews, vol. 5, no. 4, pp. 283-306, 2009.
[72] T. A. Roepke, C. Xue, M. A. Bosch, T. S. Scanlan, M. J. Kelly, and O. K. Ronnekleiv, "Genes associated with membrane-initiated signaling of estrogen and energy homeostasis," Endocrinology, vol. 149, no. 12, pp. 61136124, 2008.

[73] R. Monteiro, D. Teixeira, and C. Calhau, "Estrogen signaling in metabolic inflammation," Mediators of Inflammation, vol. 2014, 615920 pages, 2014.

[74] J. A. Engelman, J. Luo, and L. C. Cantley, "The evolution of phosphatidylinositol 3-kinases as regulators of growth and metabolism," Nature Reviews. Genetics, vol. 7, no. 8, pp. 606619, 2006

[75] G. Song, G. Ouyang, and S. Bao, "The activation of Akt/PKB signaling pathway and cell survival," Journal of Cellular and Molecular Medicine, vol. 9, no. 1, pp. 59-71, 2005.

[76] V. Duronio, "The life of a cell: apoptosis regulation by the PI3K/PKB pathway," The Biochemical Journal, vol. 415, no. 3, pp. 333-344, 2008.

[77] K. Ren, Y. J. Lu, Z. C. Mo et al., “ApoA-I/SR-BI modulates S1P/S1PR2-mediated inflammation through the PI3K/Akt signaling pathway in HUVECs," Journal of Physiology and Biochemistry, vol. 73, no. 2, pp. 287-296, 2017.

[78] A. Tsuchiya, T. Kanno, H. Nagaya, T. Shimizu, A. Tanaka, and T. Nishizaki, "PTP1B inhibition causes Rac1 activation by enhancing receptor tyrosine kinase signaling," Cellular Physiology and Biochemistry, vol. 33, no. 4, pp. 1097-1105, 2014.

[79] Q. W. Fan and W. A. Weiss, "Targeting the RTK-PI3K-mTOR axis in malignant glioma: overcoming resistance," Current Topics in Microbiology and Immunology, vol. 347, pp. 279296, 2010.

[80] W. Zhang, J. Hou, X. Yan et al., "Platycodon grandiflorum saponins ameliorate cisplatin-induced acute nephrotoxicity through the NF- $\kappa \mathrm{B}-$ Mediated inflammation and PI3K/Akt/apoptosis signaling pathways," Nutrients, vol. 10, no. 9, p. 1328, 2018.

[81] M. J. Berridge, P. Lipp, and M. D. Bootman, "The versatility and universality of calcium signalling," Nature Reviews. Molecular Cell Biology, vol. 1, no. 1, pp. 11-21, 2000.

[82] G. R. Ferrier and S. E. Howlett, "Cardiac excitationcontraction coupling: role of membrane potential in regulation of contraction," American Journal of Physiology. Heart and Circulatory Physiology, vol. 280, no. 5, pp. H1928-H1944, 2001.

[83] A. St-Denis, F. Chano, P. Tremblay, Y. St-Pierre, and A. Descoteaux, "Protein kinase C- $\alpha$ modulates lipopolysaccharide-induced functions in a murine macrophage cell line," The Journal of Biological Chemistry, vol. 273, no. 49, pp. 32787-32792, 1998.

[84] Y. Han, T. Meng, N. R. Murray, A. P. Fields, and A. R. Brasier, "Interleukin-1-induced nuclear factor- $\kappa \mathrm{B}-\mathrm{I} \kappa \mathrm{B} \alpha$ autoregulatory feedback loop in hepatocytes," The Journal of Biological Chemistry, vol. 274, no. 2, pp. 939-947, 1999.

[85] J. Siednienko, J. Nowak, P. N. Moynagh, and W. A. Gorczyca, "Nitric oxide affects IL-6 expression in human peripheral blood mononuclear cells involving cGMP-dependent modulation of NF- $\kappa$ B activity," Cytokine, vol. 54 , no. 3, pp. 282-288, 2011.

[86] E. J. Tsai and D. A. Kass, "Cyclic GMP signaling in cardiovascular pathophysiology and therapeutics," Pharmacology \& Therapeutics, vol. 122, no. 3, pp. 216-238, 2009. 
[87] E. Takimoto, "Cyclic GMP-dependent signaling in cardiac myocytes," Circulation Journal, vol. 76, no. 8, pp. 1819-1825, 2012.

[88] M. T. Bryan, H. Duckles, S. Feng et al., "Mechanoresponsive networks controlling vascular inflammation," Arteriosclerosis, Thrombosis, and Vascular Biology, vol. 34, no. 10, pp. 21992205, 2014.

[89] J. Chen, J. Green, A. Yurdagul Jr., P. Albert, M. C. McInnis, and A. W. Orr, " $\alpha v \beta 3$ integrins mediate flow-induced NF- $\kappa$ B activation, proinflammatory gene expression, and early atherogenic inflammation," The American Journal of Pathology, vol. 185, no. 9, pp. 2575-2589, 2015.

[90] D. P. Nguyen, J. Li, and A. K. Tewari, "Inflammation and prostate cancer: the role of interleukin 6 (IL-6)," BJU International, vol. 113, no. 6, pp. 986-992, 2014.

[91] K. Tominaga, T. Yoshimoto, K. Torigoe et al., "IL-12 synergizes with IL-18 or IL-1beta for IFN-gamma production from human T cells," International Immunology, vol. 12, no. 2, pp. 151-160, 2000.

[92] H. Nie, Y. Zheng, R. Li et al., "Phosphorylation of FOXP3 controls regulatory $\mathrm{T}$ cell function and is inhibited by TNF- $\alpha$ in rheumatoid arthritis," Nature Medicine, vol. 19, no. 3, pp. 322-328, 2013.

[93] P. Saiki, Y. Nakajima, L. Van Griensven, and K. Miyazaki, "Real-time monitoring of IL-6 and IL-10 reporter expression for anti- inflammation activity in live RAW 264.7 cells," Biochemical and Biophysical Research Communications, vol. 505, no. 3, pp. 885-890, 2018. 